\title{
Interpersonal synchrony: from social perception to social interaction
}

\author{
Mohamed CHETOUANI ${ }^{1}$, Emilie DELAHERCHE ${ }^{1}$, Guillaume DUMAS ${ }^{2}$, \\ David COHEN ${ }^{13}$
}

\subsection{Introduction}

Synchrony refers to individuals' temporal coordination during social interactions (Cappella, 2005). The analysis of this phenomenon is complex, requiring the perception and integration of multimodal communicative signals. The evaluation of synchrony has received multidisciplinary attention because of its role in early development (Feldman, 2003), language learning (Goldstein et al., 2003) and social connection (Harrist and Waugh, 2002). Initially, instances of synchrony were directly perceived in the data by trained observers. Several methods have been proposed to evaluate interactional synchrony, ranging from behavior micro-analysis (Cappella, 1997) to global perception of synchrony (Bernieri et al., 1988). Behavioral synchrony has now captured the interest of researchers in such fields as social signal processing, robotics and machine learning (Prepin and Pelachaud, 2011; Kozima et al., 2009).

In this chapter, we focus especially on description and definition of synchrony for the development of computational models. The chapter begins with a review of evidences of interpersonal synchrony from different research domains (psychology, clinics, neuroscience and biology). Then, we introduce a working definition of interpersonal synchrony (section 1.4). The chapter surveys evaluation models and methods from the literature of psychology (section 1.5) and social signal processing (section 1.6). Finally, the chapter discusses a number of challenges that need to be addressed (section 1.7).

1 Institut des Systèmes Intelligents et de Robotique, CNRS UMR 7222 , Université Pierre et Marie Curie, 75005 Paris, France (e-mail: mohamed.chetouani@upmc.fr)

2 Center for Complex Systems and Brain Sciences, Florida Atlantic University

3 Department of Child and Adolescent Psychiatry, Hopital de la Pitie-Salpetriere, University Pierre and Marie Curie, 75013 Paris, France 


\subsection{Non-verbal evidence of interpersonal synchrony}

Among social signals, synchrony and coordination have been considered lately (Ramseyer and Tschacher, 2010; Delaherche et al., 2012). (Condon and Ogston, 1967) initially proposed a micro-analysis of human behavior (body motion and speech intonation) and evidenced the existence of interactional synchrony, the coordination between listener's and speaker's body movements or between the listener's body movement and the speaker's pitch and stress variations. (Bernieri et al., 1988) define coordination as "... degree to which the behaviors in an interaction are non-random, patterned or synchronized in both form and timing". (Kendon, 1970) raises fundamental questions about the condition of interactional synchrony arousal and its function in interaction. When he synchronizes with the speaker, the listener demonstrates his ability to anticipate what the speaker is going to say. This way, he gives feedback to the speaker and smoothens the running of the encounter.

In the "double video setting", several teams manipulated the timing of exchanges between mother and baby by alternating live and pre-recorded exchanges (Nadel et al., 1999). They showed that in the pre-recorded sessions the child showed more negative signs (anger or distress manifestations, cries) and that when they came back to the live exchanges, the positive signals (gazes toward the mother, smiles...) were restored. In these experiments, they demonstrated expectancies for synchronized and contingent exchanges with the social partner (here the mother), since two months old. The key role of synchrony was also found at early age in more natural early interaction such as home breast feeding (Viaux-Savelon et al., 2012). In (Saint-Georges et al., 2011), we investigated early signs of autism by modeling child's development with an interpersonal synchrony point of view. Regarding synchrony, the main results show that (i) parents seemed to feel weaker interactive responsiveness and mainly weaker initiative from their infants; and (ii) parents increasingly tried to supply soliciting behaviours and touching.

As part of the social signals, interpersonal coordination is a signal of great importance to evaluate the degree of attention or engagement between two social partners. It is often related to the quality of interaction (Chartrand and Bargh, 1999), cooperation (Wiltermuth and Heath, 2009) or entitativity (Lakens, 2010). Finally, its assessment constitutes the first step in prospect of equipping a social robot with the ability to anticipate a human partner reaction and enter in synchrony with him (Michalowski et al., 2009; Prepin and Gaussier, 2010; Boucenna et al., 2014). 


\subsection{Biological evidence of interpersonal synchrony}

Concerning the development of social interaction, it is important to highlight the major role of synchrony of rhythms in bonding. Thus, Guedeney et al., 2011 emphasize the importance of synchronization between infant and parental rhythms in very early social interaction and socio-emotional development, from biological rhythms during pregnancy to later exchange between caregiver and child.

Synchrony between partners has been correlated with biological markers. Correlation at biological levels has also been found. In (Feldman, 2007), a biobehavioral synchrony model is introduced on the basis of investigations of synchrony through physiological signals (e.g. ECG, skin conductance) and behaviors during parent-infant interactions.

Naturally occurring variations in maternal behavior are associated with differences in estrogen-inducible central oxytocin receptors, which are involved in pro-social behaviors (Champagne et al., 2001). Oxytocin appears to enhance both maternal/paternal as well as affiliative behaviors in humans and is considered as the bonding hormone (Weisman et al., 2012).

(Dumas et al., 2010) use hyper-scanning recordings to examine brain activity, including measures of neural synchronization between distant brain regions of interacting individuals through a free exchange of roles between the imitator and the model. Their study was the first to record dual EEG activity in dyads of subjects during spontaneous nonverbal interaction. Five female-female pairs and 6 male-male pairs were scanned. They showed that interpersonal hand movements were correlated with the emergence of synchronization in the brain's alpha-mu band (an area involved in social interaction (Perry et al., 2010)) between the right centro-parietal regions.

Rhythm, synchrony and emotion are increasingly being viewed by developmental psychologists as key aspects of appropriate early interaction (Feldman, 2007; Saint-georges et al., 2013; Weisman et al., 2013)

\subsection{Proposed definition}

Synchrony is the dynamic and reciprocal adaptation of the temporal structure of behaviors between interactive partners. Unlike mirroring or mimicry, synchrony is dynamic in the sense that the important element is the timing, rather than the nature of the behaviors. As noted in (Ramseyer and Tschacher, 2006), the distinction between synchrony and mirroring can be unclear; these phenomena are not disjunctive and can often be observed simultaneously. 
As described in (Harrist and Waugh, 2002), synchrony requires a (1) maintained, (2) shared focus of attention, (3) temporal coordination, and (4) contingency. Computational models of synchrony need, if not all, most of theses ingredients. And the main problem is that each of them is ambiguous and requires investigations. However, taking into account advances in various fields: computational linguistics, social signal processing and social robotics or either virtual agents.

\subsection{Non-computational methods of synchrony assessment}

Several non-computational methods have been proposed to evaluate interpersonal synchrony, ranging from behavior micro-analysis to global perception of synchrony. Behavioral coding methods propose evaluating the behavior of each interactional partner on a local scale. These methods require the use of computer-based coding (e.g., Observer or Anvil (Kipp, 2008)) and trained raters. Various category and time scales can be used for coding. Generally, a measure of synchrony is deduced from the covariation of the annotated behaviors. The codes can be either continuous (speed of a gesture) or categorical (type of gesture). (Cappella, 2005) synthesized the three crucial questions to be addressed when conducting an interaction study: "what to observe (coding), how to represent observations (data representations) and when and how frequently to make the observations (time)".

Behavioral coding methods are time-consuming and tedious with regard to the training of observers, the number of behaviors coded and the duration of the video files to be coded, particularly for longitudinal studies. (Cappella, 1997) and (Bernieri et al., 1988) proposed an alternative to behavior microanalysis: the judgment method. In their studies, they investigated the use of human raters to evaluate video clips of infants interacting with their mothers. Raters judge for simultaneous movement, tempo similarity and coordination and smoothness on a longer time scale using a Likert scale. Cappella showed that untrained judges were consistent with one another and reliably judged the synchrony between partners (Cappella, 1997).

Non-computational methods suffer serious drawbacks. Within the tedious task of coding, segmenting and annotating behaviors can be confusing: when does a behavior start, when does it end, how should it be labeled? Often, the annotator makes trade-off because no label accurately describes what he observes. The judges' reliability in assessing such a subjective and complex construct is also questionable, and no general framework for synchrony assessment has been accepted to date. A method was recently proposed to convert the judgments of multiple annotators in a study on dominance into 
a machine-learning framework (Chittaranjan et al., 2011). Finally, conversational partners are often studied individually when coding. Thus, it is particularly difficult to recreate the dynamic and interpersonal aspects of social interaction manually and after coding. Nonetheless, annotation and judgment methods are essential in proposing automatic systems for synchrony assessment and testing their performance. Currently, no automatic systems modeling synchrony using real interaction data are free from annotation.

Annotation is mainly used in two different manners. First, annotation is used to train automatic systems to model and learn communication dynamics (see section 1.6.1). These studies often rely on behavioral coded databases. Second, another set of studies intends to measure the degree of synchrony between dyadic partners with unsupervised methods. In these studies, the measure of synchrony is not validated per se but is judged by its ability to predict an outcome variable that has been manually annotated, often using judgment methods. The outcome variable can be friendship (Altmann, 2011), conflicting situations (Altmann, 2011), success in psychotherapy (Ramseyer and Tschacher, 2011), etc.

\subsection{Fully automatic measures of synchrony}

To exploit synchrony cues in human-machine interaction, automatic techniques can be used to capture pertinent social signals and assess movement synchrony in human-human interactions. This studies aim at measuring the degree of similarity between the dynamics of the non-verbal behaviors of dyadic partners. The goals of these studies are generally divisible into two categories: (a) compare the degree of synchrony under different conditions (e.g., with or without visual feedback) (Shockley et al., 2003; Varni et al., 2010) and (b) study the correlation between the degree of synchrony and an outcome variable (e.g., friendship, relationship quality) (Altmann, 2011; Ramseyer and Tschacher, 2011).

The first step in computing synchrony is to extract the relevant features of the dyad's motion with motion-tracking devices (Ashenfelter et al., 2009), image-processing techniques (tracking algorithms, image differencing) (Delaherche and Chetouani, 2010; Varni et al., 2010) or physiological sensors (Varni et al., 2010). After extracting the motion features, a measure of similarity is applied. Correlation is the most commonly used method to assess interactional synchrony (Altmann, 2011; Ramseyer and Tschacher, 2011). A time-lagged cross-correlation is applied between the movement time series of the interactional partners using short windows of interaction. Another method to assess the similarity of motion of two partners is recurrence anal- 
ysis (Richardson et al., 2008). Recurrence analysis assesses the points in time that two systems show similar patterns of change or movement, called "recurrence points". Spectral methods constitute an interesting alternative to temporal methods when dealing with rhythmic tasks. Spectral methods measure the evolution of the relative phase between the two partners as an indication of a stable time-lag between them (Oullier et al., 2008; Richardson et al., 2007). Spectral methods also measure the overlap between the movement frequencies of the partners, called cross-spectral coherence (Richardson and Dale, 2005; Richardson et al., 2007; Delaherche and Chetouani, 2010) or power spectrum overlap (Oullier et al., 2008).

A critical question when attempting to detect dependence relationships between features is where the boundary between scores indicating significant and insignificant synchrony should be. A well-spread method consists of applying surrogate statistical testing (Richardson and Dale, 2005; Ashenfelter et al., 2009; Sun et al., 2011; Delaherche and Chetouani, 2010). Video images of dyadic partners are isolated and re-combined in a random order to synthesize surrogate data (pseudo-interactions). Synchrony scores are assessed using the original and surrogate datasets. The synchrony scores on the surrogate dataset constitute a baseline for judging for the dyad's coordination. Fully automatic measures of movement synchrony are subject to several criticisms in the context of studying naturalistic interaction data. First, the measures provided by these methods are mostly global and do not shed light on what happened locally during the interaction; they do not provide a local model of the communication dynamics. Second, the importance of speech and multimodality is often concealed in these methods.

\subsubsection{Machine understanding of interpersonal synchrony}

Given these criticisms, many in the field adopted the alternative practice of modeling the timing and occurrence of higher-level behavioral events such as smiles, head gestures, gazes and speaker changes. These behavioral events can be either extracted from a human-annotated database or predicted from low-level signals automatically extracted from data. These methods arise from a great interest in identifying the dynamical patterns of interaction and characterizing recurrent interpersonal behaviors.

Machine learning methods offer an interesting framework for the exploration of interactive behaviors. A key challenge is proposing models with the content and temporal structure of dyadic interactions. Various sequential learning models, such as Hidden Markov Models (HMMs) or Conditional Random Fields (CRFs), are usually used to characterize the temporal 
structure of social interactions. Messinger et al. employ related techniques for the understanding of communicative development, which is characterized by mutual influences during interaction: infants and parents influence and respond to one another during communication (Messinger et al., 2010). In (Mahdhaoui and Chetouani, 2011), an integrative approach is proposed to explicitly consider the interaction synchrony of behaviors. The model is applied to the characterization of parent-infant interactions for differential diagnosis: autism (AD), intellectual disability (ID) and typical development (TD). The authors estimate transitions between behaviors of the infant and the parent by analyzing behaviors co-occurring in a 3s window.

Among interpersonal behaviors, the prediction of turn-taking and backchannels has been largely studied in the perspective of building fluent dialog systems. The central idea is to develop "predictive models of communication dynamics that integrate previous and current actions from all interlocutors to anticipate the most likely next actions of one or all interlocutors" (Ozkan et al., 2010). The purpose of the turn-taking prediction is to accurately predict the timing between speaker transitions and the upcoming type of utterance (speaker holding the floor, speaker changes) as it occurs in humanhuman interactions (Ward et al., 2010). Back-channel behavior assures the speaker that the listener is paying attention and is in the same state in the conversation (Thorisson, 2002). Several teams have investigated how the speaker behavior triggered listeners' back-channels (Morency et al., 2008; Huang et al., 2011; Gravano and Hirschberg, 2009; Al Moubayed et al., 2009).

\subsection{Conclusions and main challenges}

Several questions regarding the dimension and perception of synchrony remain to be explored. These questions are fundamental to the development of an automatic model to assess synchrony.

The first issue relates to the nature of synchrony: is synchrony an all-ornone condition (synchronous vs. non-synchronous)? Is synchrony a continuous or a discrete notion? Or can dyadic interaction can approach or move away from synchrony (Harrist and Waugh, 2002)? Most current sources suggest that synchrony varies over the course of interaction, being stronger at the beginning and the ending of an exchange (Kendon, 1970) or at moments of particular engagement (Campbell, 2009). Feldman operationalizes synchrony as the degree to which the partners change their affective behavior in reference to one another and obtains a number ranging between 0 and 1 (Feldman, 2003). When addressing the matter of movement synchrony and 
its relation to perceived entitativity, Lakens observed that objective differences in movement rhythms were linearly related to ratings of perceived entitativity (Lakens, 2010). A recent study showed that the perception of coordination was more unanimous when coordination was very high or very low. However, judges were not reliable when judging dyads with "medium" coordination (Delaherche and Chetouani, 2011).

The second issue relates to the multiple scales of interpersonal synchrony. As previously described, there are evidences of interpersonal synchrony at different levels: behavioral, neural and physiological. One of the major challenge is to propose frameworks dealing with these different levels (Kelso et al., 2013; Chatel-Goldman et al., 2013). This will require specific tools and protocols in order to acquire, process and model various signals. In addition, interpersonal synchrony has been found at different timescales: ranging from milli-seconds to minutes. Social signal processing approaches should now deal with multi-scale situations using various sources of information. In (Weisman et al., 2013) it is described a first approach to analyze the effect of oxytocin during parent-infant interaction. Understanding these mechanisms will help to propose objective evaluation of interpersonal synchrony and more generally be of great benefit for social signal processing in terms of low-resolution brain scanning (Pentland et al., 2009).

The third issue is related to acquisition and annotation of databases. Indeed, the definition of coordination is wide and different dimensions of coordination can be analyzed. Several works have shown that the similarity measures do not always predict the degree of coordination perceived by annotators. Which begs the question : what are the signals received by the annotators annotate when partners are coordinated? These questions relate to definitions and dimensions of interpersonal synchrony. In response, a collaboration with psychologists seems essential. The question of the corpus is also crucial. Like in other related domains, i.e. affective computing, real-life, annotated and publicly distributed databases were a breakthrough that allowed researchers to propose new relevant models (e.g. continuous models of emotions). Indeed, define a research protocol, collect interaction data and annotate them is a long process. In addition, these baselines would compare the performance of different systems. Until (Sun et al., 2011) recent contribution of their mimicry database, no publicly available annotated corpus were dedicated to the detection of synchrony. We can hope that this effort will benefit the field, aiding engineers in their work to develop new algorithms, skipping the data collection and annotation phases.

The fourth issue is related to machine understanding of interpersonal synchrony. Most of studies investigate interpersonal synchrony through similar- 
ity measures (ranging from correlation to recurrence analysis) in relation to variables such as pathological groups, success of interaction... Very few studies are proposing predictive approaches: evaluation against ground truth by using traditional machine learning metrics (Petridis et al., 2013; Michelet et al., 2012; Delaherche et al., 2013). Reasons are multiple and include obviously lack of databases. Definitions may also help to propose relevant models. For instance, in (Delaherche et al., 2013), imitation is considered as unsupervised action recognition problem, where the idea is to detect similar actions independently on the nature of actions performed by the partner.

The last issue relates to the identification of applications. Automatic characterization of interpersonal synchrony might be of great interest in psychology. Such methods could provide automatic and objective tools to study interactive abilities in several psychiatric conditions, such as depression and autism. Although few studies are currently available in this specific field, they appear to be very promising: couple therapy (Lee et al., 2011), success in psychotherapy (Ramseyer and Tschacher, 2011), mother-infant interaction (Cohn, 2010). Another great potential lies in the opportunity to build robots or virtual agents with interactive abilities (Gratch et al., 2007; Al Moubayed et al., 2009; Prepin and Pelachaud, 2011; Boucenna et al., 2014).

\section{Acknowledgments}

This work was supported by the UPMC "Emergence 2009" program, the European Union Seventh Framework Programme under grant agreement n288241, the the Agence Nationale de la Recherche (SAMENTA program: SYNED-PSY). This work was performed within the Labex SMART supported by French state funds managed by the ANR within the Investissements d'Avenir programme under reference ANR-11-IDEX-0004-02. 


\section{References}

Al Moubayed, S., Baklouti, M., Chetouani, M., Dutoit, T., Mahdhaoui, A., Martin, J. C., Ondas, S., Pelachaud, C., Urbain, J., and Yilmaz, M. 2009. Generating Robot/Agent backchannels during a storytelling experiment. Robotics and Automation, 2009. ICRA '09. IEEE International Conference on, 3749-3754.

Altmann, U. 2011. Studying Movement Synchrony Using Time Series and Regression Models.

Ashenfelter, K. T., Boker, S. M., Waddell, J. R., and Vitanov, N. 2009. Spatiotemporal symmetry and multifractal structure of head movements during dyadic conversation. J Exp Psychol Hum Percept Perform, 35(4), 1072-91.

Bernieri, F.J., Reznick, J.S., and Rosenthal, R. 1988. Synchrony, pseudo synchrony, and dissynchrony: Measuring the entrainment process in mother-infant interactions. Journal of Personality and Social Psychology, 54(2), 243-253.

Boucenna, S., Anzalone, S., Tilmont, E., Cohen, D., and Chetouani, M. 2014. Learning of social signatures through imitation game between a robot and a human partner. In: IEEE Transactions on Autonomous Mental Development (to appear).

Campbell, N. 2009. An audio-visual approach to measuring discourse synchrony in multimodal conversation data. Pages 2159-2162 of: Interspeech.

Cappella, J. 2005. Coding Mutual Adaptation in Dyadic Nonverbal Interaction.

Cappella, J.N. 1997. Behavioral and judged coordination in adult informal social interactions: vocal and kinesic indicators. Pers. Soc. Psychol., 72, 119-131.

Champagne, F., Diorio, J., Sharma, S., and Meaney, M. J. 2001. Naturally occurring variations in maternal behavior in the rat are associated with differences in estrogen-inducible central oxytocin receptors. Proceedings of the National Academy of Sciences, 98(22), 12736-12741.

Chartrand, T. L., and Bargh, J. A. 1999. The chameleon effect: The perceptionbehavior link and social interaction. Journal of personality and social psychology, 76(6), 893-910.

Chatel-Goldman, J., Schwartz, J.-L., Jutten, C., and Congedo, M. 2013. Nonlocal mind from the perspective of social cognition. Frontiers in Human Neuroscience, $\mathbf{7}(107)$.

Chittaranjan, G., Aran, O., and Gatica-Perez, D. 2011. Inferring truth from multiple annotators for social interaction analysis. Page 4 of: Neural Information Processing Systems (NIPS) Workshop on Modeling Human Communication Dynamics (HCD). 
Cohn, J. F. 2010. Advances in Behavioral Science Using Automated Facial Image Analysis and Synthesis. IEEE Signal Processing Magazine, 27(November), $128-133$.

Condon, W.S., and Ogston, W.D. 1967. A segmentation of behavior. Journal of Psychiatric Research, 5, 221-235.

Delaherche, E., and Chetouani, M. 2010. Multimodal coordination: exploring relevant features and measures. In: Second International Workshop on Social Signal Processing, ACM Multimedia 2010.

Delaherche, E., and Chetouani, M. 2011. Characterization of coordination in an imitation task : human evaluation and automatically computable cues. In: 13th International Conference on Multimodal Interaction.

Delaherche, E., Chetouani, M., Mahdhaoui, M., Saint-Georges, C., Viaux, S., and Cohen, D. 2012. Interpersonal Synchrony : A Survey Of Evaluation Methods Across Disciplines. IEEE Transactions on Affective Computing, 3(3), 349365 .

Delaherche, E., Boucenna, S., Karp, K., Michelet, S., Achard, C., and Chetouani, M. 2013. Social Coordination Assessment: Distinguishing between Shape and Timing. Pages 9-18 of: Multimodal Pattern Recognition of Social Signals in Human-Computer-Interaction. Lecture Notes in Computer Science, vol. 7742. Springer Berlin Heidelberg.

Dumas, G., Nadel, J., Soussignan, R., Martinerie, J., and Garnero, L. 2010. InterBrain Synchronization during Social Interaction. PLOS ONE, 5(8), e12166.

Feldman, R. 2003. Infant-mother and infant-father synchrony : the coregulation of positive arousal. Infant Mental Health Journal, 24(1), 1-23.

Feldman, R. 2007. Parent-infant synchrony and the construction of shared timing; physiological precursors, developmental outcomes, and risk conditions. The Journal of Child Psychology and Psychiatry and Allied Disciplines, 48(3-4), 329-354.

Goldstein, Michael H, King, Andrew P, and West, Meredith J. 2003. Social interaction shapes babbling: Testing parallels between birdsong and speech. Proceedings of the National Academy of Sciences of the United States of America, 100(13), 8030-8035.

Gratch, J., Wang, N., Gerten, J., Fast, E., and Duffy, R. 2007. Creating Rapport with Virtual Agents. Pages 125-138 of: IVA '07: Proceedings of the 7th international conference on Intelligent Virtual Agents. Springer-Verlag.

Gravano, A., and Hirschberg, J. 2009. Backchannel-inviting cues in task-oriented dialogue. Pages 1019-1022 of: INTERSPEECH.

Guedeney, A., Guedeney, N., Tereno, S., Dugravier, R., Greacen, T., Welniarz, B., Saias, T., and Tubach, F. 2011. Infant rhythms versus parental time: Promoting parent-infant synchrony. Journal of Physiology-Paris, 105(4-6), $195-200$.

Harrist, A.W., and Waugh, R.M. 2002. Dyadic synchrony: Its structure and function in children's development. Developmental Review, 22(4), 555-592.

Huang, L., Morency, L.-P., and Gratch, J. 2011. A multimodal end-of-turn prediction model: learning from parasocial consensus sampling. Pages 1289-1290 of: The 10th International Conference on Autonomous Agents and Multiagent Systems - Volume 3. AAMAS '11.

Kelso, J.A. S., Dumas, G., and Tognoli, E. 2013. Outline of a general theory of behavior and brain coordination. Neural Networks, 37(0), 120 - 131. 
Kendon, A. 1970. Movement coordination in social interaction: some examples described. Acta Psychologica, 32, 100-125.

Kipp, M. 2008. Spatiotemporal Coding in ANVIL. In: LREC.

Kozima, H., Michalowski, M., and Nakagawa, C. 2009. Keepon. International Journal of Social Robotics, 1, 3-18.

Lakens, D. 2010. Movement synchrony and perceived entitativity. Journal of Experimental Social Psychology, 46(5), $701-708$.

Lee, C., Katsamanis, A., Black, M. P., Baucom, B. R., Georgiou, P. G., and Narayanan, S. 2011. An Analysis of PCA-based Vocal Entrainment Measures in Married Couples, Affective Spoken Interactions. In Proceedings of InterSpeech, 3101-3104.

Mahdhaoui, A., and Chetouani, M. 2011. Understanding parent-infant behaviors using non-negative matrix factorization. Pages 436-447 of: Proceedings of the Third COST 2102 international training school conference on Toward autonomous, adaptive, and context-aware multimodal interfaces: theoretical and practical issues. Berlin, Heidelberg: Springer-Verlag.

Messinger, D. M., Ruvolo, P., Ekas, N. V., and Fogel, A/. 2010. Applying machine learning to infant interaction: The development is in the details. Neural Networks, 23(8-9), $1004-1016$.

Michalowski, M.P., Simmons, R., and Kozima, H. 2009. Rhythmic attention in child-robot dance play. In: Proceedings of RO-MAN 2009.

Michelet, S., Karp, K., Delaherche, E., Achard, C., and Chetouani, M. 2012. Automatic Imitation Assessment in Interaction. Pages 161-173 of: Human Behavior Understanding. Lecture Notes in Computer Science, vol. 7559. Springer Berlin Heidelberg.

Morency, L.-P., Kok, I., and Gratch, J. 2008. Predicting Listener Backchannels: A Probabilistic Multimodal Approach. Pages 176-190 of: Proceedings of the 8th international conference on Intelligent Virtual Agents. IVA '08. Berlin, Heidelberg: Springer-Verlag.

Nadel, J., Carchon, I., Kervella, C., Marcelli, D., and Roserbat-Plantey, D. 1999. Expectancies for social contingency in 2-month-olds. Developmental Science, 2(2), 164-173.

Oullier, O., de Guzman, G. C., Jantzen, K. J., S. Kelso, J. A., and Lagarde, J. 2008. Social coordination dynamics: Measuring human bonding. Social Neuroscience, 3(2), 178-192.

Ozkan, D., Sagae, K., and Morency, L.-P. 2010. Latent Mixture of Discriminative Experts for Multimodal Prediction Modeling. Computational Linguistics, 860868.

Pentland, Alex, Lazer, David, Brewer, Devon, and Heibeck, Tracy. 2009. Using reality mining to improve public health and medicine. Stud Health Technol Inform, 149, 93-102.

Perry, Anat, Troje, Nikolaus F., and Bentin, Shlomo. 2010. Exploring motor system contributions to the perception of social information: Evidence from EEG activity in the mu/alpha frequency range. Social Neuroscience, 5(3), 272-284.

Petridis, S., Leveque, M., and Pantic, M. 2013 (September). Audiovisual Detection of Laughter In Human Machine Interaction. Pages 129-134 of: Affective Computing and Intelligient Interaction (ACII 2013).

Prepin, K., and Gaussier, P. 2010. How an agent can detect and use synchrony parameter of its own interaction with a human? Pages 50-65 of: Esposito, 
Anna, et al. (eds), Development of Multimodal Interfaces: Active Listening and Synchrony, vol. 5967. Springer Berlin / Heidelberg.

Prepin, K., and Pelachaud, C. 2011. Shared understanding and Synchrony Emergence: Synchrony as an Indice of the Exchange of Meaning between Dialog Partners. Pages 25-30 of: ICAART2011 International Conference on Agent and Artificial Intelligence, vol. 2.

Ramseyer, F., and Tschacher, W. 2006. Synchrony: A Core Concept for A Constructivist Approach to Psychotherapy. Constructivism. The Human Sciences., 11, $150-171$.

Ramseyer, F., and Tschacher, W. 2010. Nonverbal Synchrony or Random Coincidence? How to Tell the Difference. Pages 182-196 of: Esposito, Anna, et al. (eds), Development of Multimodal Interfaces: Active Listening and Synchrony, vol. 5967. Springer Berlin / Heidelberg.

Ramseyer, F., and Tschacher, W. 2011. Nonverbal Synchrony in Psychotherapy: Coordinated Body Movement Reflects Relationship Quality and Outcome. Journal of Consulting and Clinical Psychology, 79(3), $284-295$.

Richardson, D., Dale, R., and Shockley, K. 2008. Synchrony and swing in conversation: Coordination, temporal dynamics, and communication. Oxford University Press.

Richardson, D. C., and Dale, R. 2005. Looking To Understand: The Coupling Between Speakers' and Listeners' Eye Movements and Its Relationship to Discourse Comprehension. Cognitive Science, 29(6), 1045-1060.

Richardson, Michael J., Marsh, Kerry L., Isenhower, Robert W., Goodman, Justin R.L., and Schmidt, R.C. 2007. Rocking together: Dynamics of intentional and unintentional interpersonal coordination. Human Movement Science, 26(6), $867-891$.

Saint-Georges, C., Mahdhaoui, A., Chetouani, M., Cassel, R. S., Laznik, M-C, Apicella, F., Muratori, P., Maestro, S, Muratori, F., and Cohen, D. 2011. Do Parents Recognize Autistic Deviant Behavior Long before Diagnosis? Taking into Account Interaction Using Computational Methods. PLoS ONE, 6(7), e22393.

Saint-georges, C., Chetouani, M., Cassel, R., Apicella, F., Mahdhaoui, A., Muratori, F., Laznik, M.C., and Cohen, D. 2013. Motherese in interaction: at the crossroad of emotion and cognition? (a systematic review). PLoS ONE, 8(10), e78103.

Shockley, K., Santana, M.-V., and Fowler, C. A. 2003. Mutual interpersonal postural constraints are involved in cooperative conversation. Journal of Experimental Psychology: Human Perception and Performance.

Sun, X., Truong, K., Nijholt, A., and Pantic, M. 2011 (June). Automatic Visual Mimicry Expression Analysis in Interpersonal Interaction. Pages 40-46 of: Proceedings of IEEE Intl Conf. Computer Vision and Pattern Recognition (CVPR-W'11), Workshop on CVPR for Human Behaviour Analysis.

Sun, X., Lichtenauer, J., Valstar, M., Nijholt, A., and Pantic, M. 2011. A Multimodal Database for Mimicry Analysis. Pages 367-376 of: Affective Computing and Intelligent Interaction, Part I. Lecture Notes in Computer Science, vol. 6974. Berlin, Germany: Springer Verlag.

Thorisson, Kristin R. 2002. Natural Turn-Taking Needs No Manual: Computational Theory And Model, From Perception to Action.

Varni, G., Volpe, G., and Camurri, A. 2010. A System for Real-Time Multimodal 
Analysis of Nonverbal Affective Social Interaction in User-Centric Media. Multimedia, IEEE Transactions on, 12(6), 576 -590.

Viaux-Savelon, S., Dommergues, M., Rosenblum, O., Bodeau, N., Aidane, E., Philippon, O., Mazet, P., Vibert-Guigue, C., Vauthier-Brouzes, D., Feldman, R., and Cohen, D. 2012. Prenatal Ultrasound Screening: False Positive Soft Markers May Alter Maternal Representations and Mother-Infant Interaction. PLoS ONE, 7(1), e30935.

Ward, Nigel G., Fuentes, Olac, and Vega, Alejandro. 2010. Dialog prediction for a general model of turn-taking. Pages 2662-2665 of: INTERSPEECH.

Weisman, O., Zagoory-Sharon, O., and Feldman, R. 2012. Oxytocin Administration to Parent Enhances Infant Physiological and Behavioral Readiness for Social Engagement. Biological Psychiatry, 72(12), 982 - 989.

Weisman, O., Delaherche, E., Rondeau, M., Chetouani, M., Cohen, D., and Feldman, R. 2013. Oxytocin shapes parental motion during father-infant interaction. Biology Letters, $\mathbf{9}(6)$.

Wiltermuth, S. S., and Heath, C. 2009. Synchrony and Cooperation. Psychological Science, 20(1), 1 - 5. 